\title{
Cell-Free Translation Is More Variable than Transcription
}

\author{
Fabio Chizzolini, Michele Forlin, Noël Yeh Martín, Giuliano Berloffa, Dario Cecchi, \\ and Sheref S. Mansy*(1)
}

Center for Integrative Biology (CIBIO), University of Trento, via Sommarive 9, 38123 Povo TN, Italy

\section{Supporting Information}
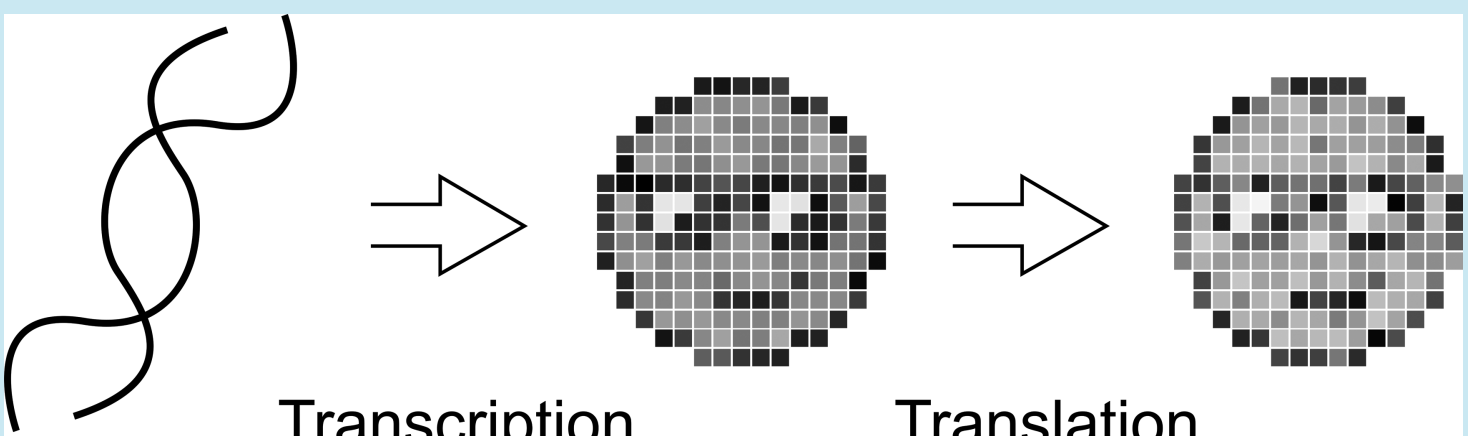

Transcription

Translation

ABSTRACT: Although RNA synthesis can be reliably controlled with different T7 transcriptional promoters during cell-free gene expression with the PURE system, protein synthesis remains largely unaffected. To better control protein levels, we investigated a series of ribosome binding sites (RBSs). Although RBS strength did strongly affect protein synthesis, the RBS sequence could explain less than half of the variability of the data. Protein expression was found to depend on other factors besides the strength of the RBS, including the GC content of the coding sequence. The complexity of protein synthesis in comparison to RNA synthesis was observed by the higher degree of variability associated with protein expression. This variability was also observed in an E. coli cell extract-based system. However, the coefficient of variation was larger with E. coli RNA polymerase than with T7 RNA polymerase, consistent with the increased complexity of E. coli RNA polymerase.

KEYWORDS: cell-free synthetic biology, transcription-translation, IVTT, PURE system, artificial cell

C ell-free expression systems have a wide-range of applications from experiments designed to gain insight into the workings of biology ${ }^{1,2}$ to the development of new technologies. $^{3-5}$ Although there are several cell-free systems to choose from, none have been well characterized with respect to the influence of different biological parts on system performance. This is a problem because complex genetic circuitry requires varying degrees of expression of each component. Perhaps the lack of cell-free standardization data reflects an initial bias toward producing maximum amounts of protein from a single gene, just as commercial vectors typically contain sequences, such as the T7 gene 10 leader sequence, to enhance the recombinant expression of protein. ${ }^{6}$ Additionally, when cellfree systems are used to screen genetic constructs for later insertion into bacteria, protein output is often controlled by adjusting the DNA template concentration rather than by controlling gene expression through sequence changes, e.g., by changing the transcriptional promoter or ribosome binding site. $^{7}$ In this case, each gene product is typically encoded on a separate piece of DNA. However, the use of separate pieces of DNA is not suitable for encapsulation in vesicles for the assembly of artificial cells nor ideal for insertion in the genome of a living cell.
Currently, there are several methods that can be used to try to build genetic circuits with a desired performance. One method is to create a computational model that can accurately predict gene expression. Such a model may incorporate the kinetic parameters of association, dissociation, and enzymatic activity as well as the influence of RNA folding. Although much progress has been made, ${ }^{8-11}$ several of the parameters governing transcription-translation are still too poorly characterized to build a model that can predict the behavior of a genetic circuit accurately with a high success rate, although the inclusion of transcriptional insulators greatly improves predictability. ${ }^{12}$ Other kinds of computational models do not directly consider the biophysical process of gene expression but instead attempt to predict gene expression through summation of the activity of individually characterized biological parts. Instead of relying on a computational model, a library of DNA molecules each containing a different mixture of biological parts can be assembled and then screened for activity. ${ }^{13,14}$ One downside of this approach is that only a small subset of all the potential sequences can be practically tested in the laboratory, decreasing the likelihood of identifying a construct with the

Received: September 6, 2016

Published: January 19, 2017 


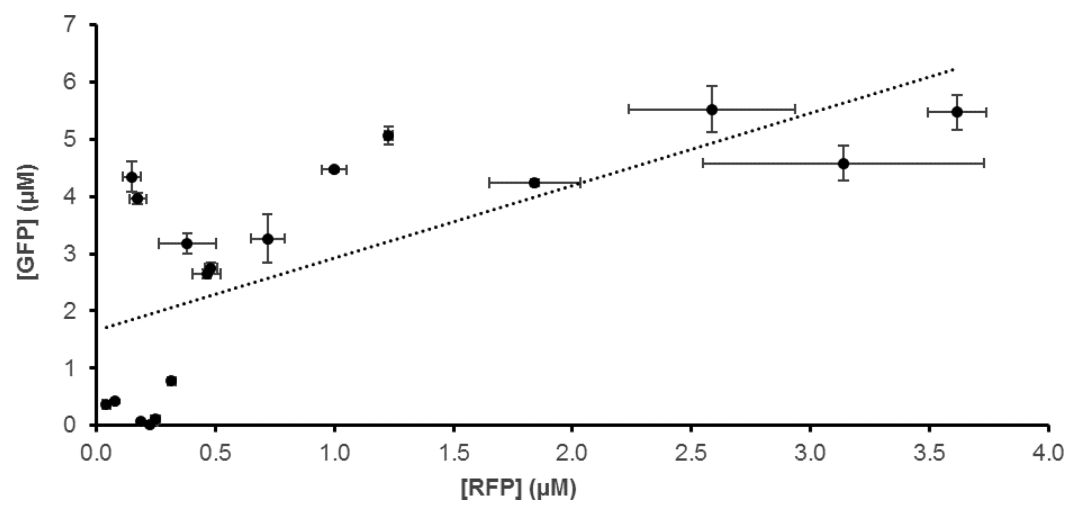

Figure 1. Expression of single gene constructs encoding RFP and GFP do not correlate well. Each point represents a template DNA containing a different ribosome binding site. Reactions were performed at $37^{\circ} \mathrm{C}$ for $6 \mathrm{~h}$. The correlation was 0.61 . The different ribosome binding sites employed are listed in Table S1. Sequence details can be found in FC001 (GFP) and FC002 (RFP) of the Supporting Information.

sought after behavior. Additionally, the assembly and testing of the library of sequences incurs significant cost and time. Both computational and wet lab screening methods frequently depend on the use of biological parts that were previously characterized by assessing the expression of a fluorescent protein. The assumption is that the substitution of the fluorescent protein by a gene of interest will be largely innocuous to system performance. For the time being, a more reasonable approach may be to exploit computational methods to identify a small subset of potential sequences with the desired behavior followed by screening in the laboratory.

Previously, we explored the influence of genetic organization $^{15}$ and T7 transcriptional promoter strength ${ }^{16}$ on cell-free expression with the protein synthesis using the recombinant elements (PURE) ${ }^{17}$ system. The data revealed that RNA but not protein concentrations were easily tuned with transcriptional promoter strength with a transcription-translation system that depended on T7 RNA polymerase. It thus seemed that the use of different ribosome binding sites would be more amenable to controlling protein synthesis because the RBSribosome complex is initially mediated by known base pairing interactions that directly correlate with the strength of interaction. ${ }^{18,19}$ Although a few reports have exploited the RBS to modulate gene expression in vitro, ${ }^{20,21}$ a systematic investigation with the PURE system ${ }^{17}$ is lacking. Here, we investigated the influence of different ribosome binding sites on gene expression with the PURE system. Unlike the strength of the T7 RNA polymerase, the RBS greatly impacted protein synthesis but not in a predictable manner. The activity of the RBS was strongly influenced by the sequence and thus the structure of the mRNA. To ensure that data from different sets of experiments were comparable, we assessed the variability in transcription and translation. Although the noise associated with cell-free expression in compartmentalized (i.e., water-in-oil emulsion droplets, lipid vesicles, or polydimethylsiloxane containers) low-copy-number systems has been well characterized, ${ }^{2,22,23}$ the variability from reactions under bulk, noncompartmentalized conditions has not been reported. We found that the variability in protein synthesis was larger than for RNA synthesis for single gene constructs. The variability increased for cascading reactions, and a computational model that included parameters for variability helped to screen genetic constructs for a subset with desired activity.

\section{RESULTS AND DISCUSSION}

Ribosome Binding Sites Strongly but Unreliably Control Gene Expression. Eighteen different ribosome binding sites were designed and tested (Table S1). The sequence of the ribosome binding site (RBS) was varied according to three variables, including the number of base pair interactions between the ribosome and the RBS, the position of the base pair interactions within the RBS, and the nucleotide composition of the nonbase pairing positions within the RBS. Thirteen of these sequences were generated using a D-criterion optimal statistical design of the experimental methodology ${ }^{24}$ using a multiple linear regression model (expressed RFP = number of base pairing interactions with the RBS + location of the base pairing interactions + identity of the nonbase paired positions), so that few sequences could be used to efficiently explore the sequence space of the RBS. The remaining five sequences were taken from previously tested constructs. ${ }^{15,16}$

The set of different ribosome binding sites was tested within a genetic construct encoding a red fluorescent protein (RFP) and with a purified, E. coli-based transcription-translation system containing T7 RNA polymerase (i.e., the PURE system) ${ }^{17}$ Contrary to what was previously observed with a series of T7 transcriptional promoters, ${ }^{16}$ the set of ribosome binding sites gave a well-dispersed distribution of expressed protein concentrations (Figure S1). However, a statistical model built with data from the expression of the $13 \mathrm{D}$-criterion optimally statistically designed sequences explained less than $50 \%$ of the variability of the data (adjusted $R^{2}=0.49$; $p$-value $=$ 0.03). Most of the explained variability came from the correlation (Pearson correlation $=0.5$ ) between RFP expression and the number of potential base pairing interactions between the RBS and the ribosome (Figure S2a), whereas the location of the base paired positions within the RBS and the identity of the nonbase paired positions had no discernible effect on the expression of RFP. This suggested that something other than ribosome binding was also significantly impacting expression. To determine whether the sequence of the encoded gene influenced the activity of the ribosome binding site, we tested the same set of ribosome binding sites within a construct encoding green fluorescent protein (GFP) (Figure S1). The correlation between the GFP and RFP data was 0.61 for the same set of 13 sequences (Figure 1), indicating that although a significant correlation existed, data from the expression of one protein could not be used to reliably predict the expression of the other protein. The correlation between 
a)

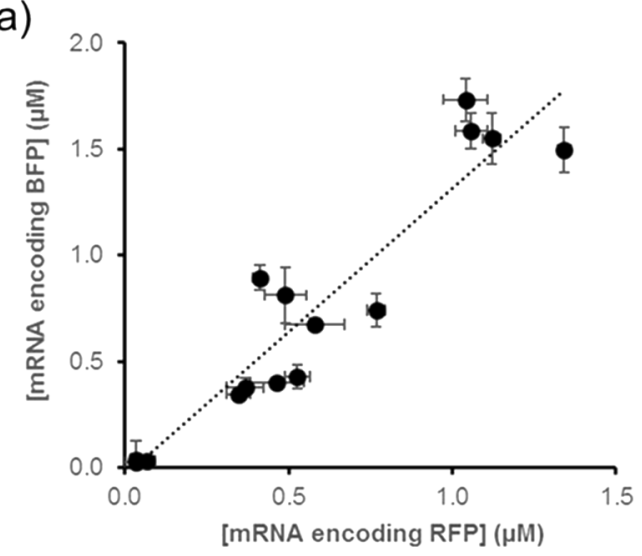

c)

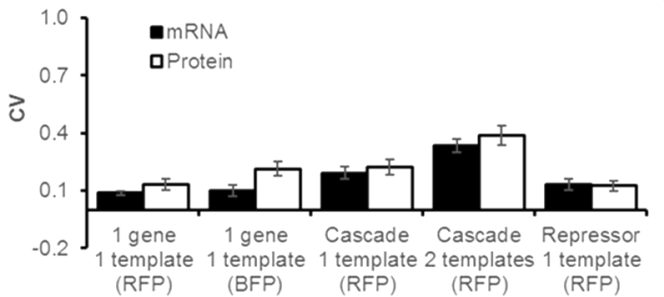

b)

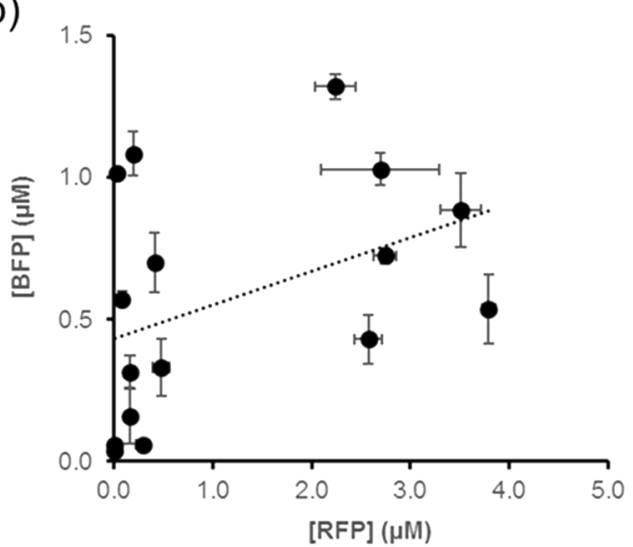

d)

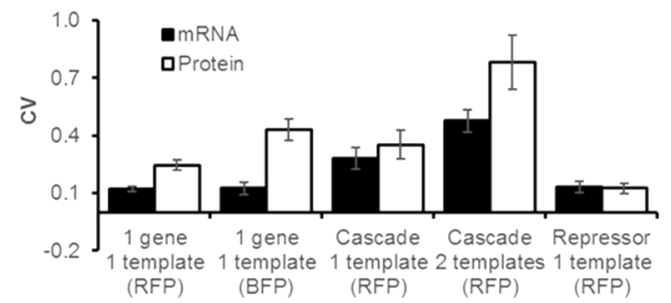

e) 1 gene

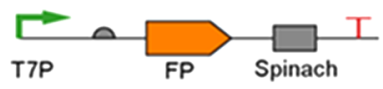

Cascade

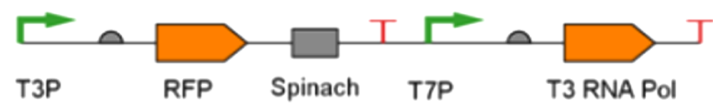

Repressor

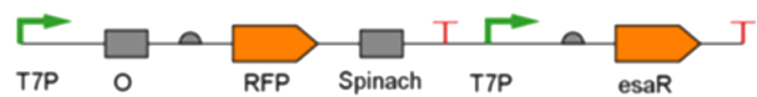

Figure 2. Consistency of transcription and translation in PURE system reactions. (a) Transcriptional promoters predictably control transcription with consistency across different template sequences without influence of the ribosome binding site, whereas (b) the same constructs show significant differences in protein synthesis. Each data point in panels a and $b$ represent the transcription-translation of a different genetic construct with a different combination of transcriptional promoter and RBS. Sequence information and RNA and protein yields can be found in Table S5 and Figure S6. Single (c) and multiple (d) batch transcription-translation reactions show more variability for translation than for transcription. The coefficient of variation (CV) was calculated for constructs encoding either one or two genes (labeled Cascade and Repressor) for reactions containing only one template or two. All of the templates encoded RFP except for one, which encoded BFP. (e) Single template versions of each genetic construct used in panel c are shown. All of the genetic constructs contained the LS1 $5^{\prime}$-UTR for the expression of the reporter gene, i.e., a fluorescent protein followed by Spinach. In the Cascade and Repressor genetic circuits, the LS3 5'-UTR was employed for the expression of T3 RNA polymerase and EsaR, respectively. The T7 transcriptional promoter (T7P), T3 transcriptional promoter (T3P), fluorescent protein (FP, representing either RFP or BFP), Spinach aptamer (Spinach), and the binding site for EsaR (O) are all labeled. Sequence details can be found under pFC013A, pDC051A, FC025-FC027, and FC032 in the inventory of composable elements (ICE).

the expression of GFP to the number of base pairing interactions with the RBS was 0.87 (with respect to the 13 D-optimal sequences) (Figure S2b), which is significantly higher than for the expression of RFP. Because the sequences of these two genes greatly differed (48\% identity), it seemed likely that the folding of the mRNA or the codon context strongly influenced protein expression, as previously noted. ${ }^{25-27}$ No correlation was found between gene expression and the position of the base pairing interactions within the RBS nor the composition of the nonbase pairing positions within the RBS. Taken together, RBS sequences significantly impacted protein expression, as expected, but the effects were not consistent between different genetic sequences, likely reflecting the influences of competing intramolecular base pairing interactions.
The 5'- and 3'-Untranslated Region (UTR) Influence Protein Expression. To more rapidly screen genetic constructs, we used linear PCR product DNA templates. Because the incorporation of a transcriptional terminator at the $3^{\prime}$-end of mRNA has been found to increase the expression of protein, ${ }^{28,29}$ we investigated the effect of the $3^{\prime}$-UTR on the expression levels of GFP and RFP. The tested DNA templates either ended directly with a stop codon (TAA) or a sequence that encoded a $56 \mathrm{bp}$ sequence of low folding stability, a $91 \mathrm{bp}$ sequence of low folding stability, the bacteriophage T7 T $\phi$ terminator (153 bp), a Spinach aptamer sequence (171 bp), the $\mathrm{T} \phi$ terminator embedded in a longer sequence ( $175 \mathrm{bp})$, and a sequence containing both the Spinach aptamer and the $\mathrm{T} \phi$ terminator $(367 \mathrm{bp})$ (Table S2). Protein expression was the lowest from constructs that lacked a $3^{\prime}$-UTR (Figure S3), as expected. ${ }^{28,29}$ Longer $3^{\prime}$-UTR sequences increased protein yield 
up to approximately $90 \mathrm{bp}$ in length at which point expression levels were 26- and 12-fold larger for RFP and GFP, respectively, than constructs without a $3^{\prime}$-UTR. There was not a strong dependence on whether the $3^{\prime}$-UTR contained a transcriptional terminator, a random sequence, or an aptamer domain (10 and $17 \%$ variance for RFP and GFP, respectively). To confirm that the $3^{\prime}$-UTR was only important for the terminal gene of an operon, we constructed two gene operons encoding both GFP and RFP. The inclusion of the T $\phi$ terminator within the $3^{\prime}$-UTR increased protein synthesis from the second but not the first gene of the operon (Figure S4).

Next, the influence of the $5^{\prime}$-UTR (excluding the ribosome binding site) on gene expression was investigated to identify sequences with similar effects on gene expression to simplify the analysis of other regions of the genetic constructs. Five different 5'-UTR sequences, including four randomly generated sequences lacking strong structural elements (LS1-LS4) and one sequence (LS5) generated by the RBS calculator, ${ }^{30}$ were tested (Table S3). Of these five sequences, the different $5^{\prime}$ UTR sequences resulted in different amounts of RNA (based on the fluorescence of the Spinach aptamer) and protein (based on the fluorescence of RFP) synthesis with three 5'-UTR sequences (LS1-LS3) giving more similar protein output (Figure S5). The influence of each of these three 5'-UTRs was also similar across different coding sequences, including those of GFP, RFP, and a blue fluorescent protein (BFP). However, the expression of RFP was lower with LS2 than with the other fluorescent proteins (e.g., the final RFP concentration was $44 \%$ lower than that of GFP with LS2).

Variability in Protein Synthesis Outweighs Variability in RNA Synthesis. Four T7 transcriptional promoters and four ribosome binding sites were then selected to make 16 different combinations of genetic constructs (Tables S4 and S5). Each DNA template contained an LS1 leader sequence and a $\mathrm{T} \phi$ terminator sequence. For both RFP and BFP, the relative transcriptional promoter strength gave predictable distributions of mRNA concentrations regardless of the strength of the RBS (Figure 2a, Figure S6). This is consistent with our previous data on a much larger set of T7 transcriptional promoters with a consistent RBS. ${ }^{16}$ Constructs encoding GFP were not assessed because of spectral overlap between GFP and the encoded Spinach aptamer used to quantify the mRNA. The influence of the RBS on the expressed protein concentration again showed poor correlation between constructs encoding RFP, BFP, and GFP for all four transcriptional promoters (Figure 2b, Figure S7).

To better assess the variability (or repeatability) of transcription-translation reactions, we measured the coefficient of variation (also called relative standard deviation) for both triplicates run on the same day with transcription-translation machinery from the same batch (total experiments $=3$ ) and on three different days with three different batches of transcriptional-translation machinery (total experiments $=9$ ). The weakest transcriptional promoters were not used for these experiments. The one batch experiments showed larger variability in expressed protein than expressed RNA for both RFP (48\% greater variability)- and BFP (116\% greater variability)-encoding constructs (Figure 2c). The multiple batch experiments did not greatly increase the coefficient of variation of transcription, whereas the coefficient of variation of translation increased by $89 \%$ for RFP and by $101 \%$ for BFP when comparing multiple batch to single batch reactions (Figure 2d). The coefficient of variation of experiments run on the same day from a single batch of transcription-translation machinery reflected, in part, error arising from the assembly of the reaction. The data collected over multiple days were additionally influenced by batch-to-batch variability of transcription-translation solutions. The low coefficient of variation of transcription for both genetic constructs was consistent with the fact that in the PURE system only a single protein is required to synthesize mRNA and that the DNA templates possess a consistent structure regardless of sequence, i.e., double stranded DNA typically assumes a b-form $\alpha$-helix under physiological conditions. Conversely, the increased coefficient of variation observed for translation was consistent with the large number of RNA and protein molecules required to synthesize protein. Furthermore, the folding of the RNA template is sequence dependent and would be expected to affect the efficiency of translation, as observed when comparing RFP and BFP synthesis. In fact, although the coefficient of variation was within $15 \%$ for the transcription of RFP and BFP, the coefficient of variation was 65 and $75 \%$ greater for the translation of BFP than RFP for one batch and multiple batch experiments, respectively. This is consistent with the lower correlations observed above for the synthesis of different proteins when compared to the synthesis of different mRNAs. To determine whether the increase in variability for transcription was dependent on the RNA polymerase or reflected a specific feature arising from the PURE system, we repeated the measurements with $\mathrm{T} 7$ and E. coli transcriptional promoters in an $E$. coli cell extract. The coefficient of variation was 5-fold larger for translation than for transcription RNA polymerase and 1.9-fold larger with E. coli RNA polymerase (Figure S8). The coefficient of variation was 10.4-fold larger for transcription with E. coli RNA polymerase than with T7 RNA polymerase, consistent with the fact that E. coli RNA polymerase is composed of multiple, separately encoded protein subunits.

The variability of transcription-translation was confirmed by assembling transcription-translation reactions in a 1536-well plate to construct a pixelated image. In this way, the clarity of the image would visually reveal the variability of RNA (based on the fluorescence of the Spinach aptamer) and protein (based on the fluorescence of RFP) synthesis. A 196 pixel version of the yin-yang symbol was designed by exploiting genetic constructs that would give rise to four different color intensities based on RNA and protein synthesis levels. Some of the wells that contained the same genetic construct also contained different batches of transcription-translation machinery. Because each color intensity was represented by at least 57 contiguous pixels, variability was easily observed visually. Consistent with the data presented above, the RNA-based image was much clearer than the protein-based image (Figure S9), confirming that RNA synthesis was less variable than protein synthesis.

A Simple Computational Model that Includes Parameters for Variability Can Reasonably Predict Cell-Free Expression. A generalization of the computational model described by Stogbauer et al. ${ }^{8}$ was built to serve as a predictive tool for the design of more complex genetic circuits in cell-free systems (Figure S10). In this model, the resources needed for transcription and translation were grouped separately. However, parameters for the strength of the T7 transcriptional promoter and the RBS were also included. ${ }^{16}$ Additionally, two noise parameters were incorporated. The first noise parameter was meant to address the error that was observed from the 
single batch experiments by allowing the DNA template concentration to fluctuate by $\pm 5 \%$. The second noise parameter approximated the error observed from the multiple batch experiments by allowing the concentrations of components of the PURE system to vary by $\pm 10 \%$. Kinetic parameters were inferred from a subset of experiments with different combinations of $\mathrm{T} 7$ transcriptional promoters and RBS sequences (Figure S7). To address the absolute difference in RFP, GFP, and BFP expression, we inferred the parameters for the kinetics of transcription and translation $\left(r_{\text {rna prod }}\right.$ and $r_{\text {prot_prod, }}$ respectively) as well as the parameter describing protein maturation ( $\left.r_{\text {prot_mat }}\right)$ for each protein. The final inferred parameters, along with the starting concentrations of the molecular components and the parameters accounting for noise, were also included (Tables S6-S8). The resulting model fit well the experimental data (Figure S11).

Because a wide-range of colors can be made by mixing different intensities of red, blue, and green colors together, we wondered if genetically encoded colored pictures could be produced by expressing different amounts of RFP, BFP, and GFP or if the variability in protein expression would interfere with the predictable formation of new colors. The kinetic model was used to identify genetic constructs that could be assembled into a 25 pixel RGB color triangle. Each well contained three different DNA templates at the same concentration, each encoding a different fluorescent protein. The model was used to indicate which $\mathrm{T} 7$ transcriptional promoter and which E. coli ribosome binding site could be used to produce 25 different colors. As seen in Figure 3, the assembled reactions produced a color triangle that closely matched the prediction, and the variability associated with the expression of three different proteins was low enough to allow for the development of the image. The average absolute differences between the predictions and the experimental proportions for the three fluorescent proteins were 32, 11, and $17 \%$ for RFP, GFP, and BFP, respectively. The largest difference between the prediction and the measured protein expression was associated with RFP, which was overestimated by the model. Under the experimental conditions employed, photobleaching did not significantly impact the measurements (Figure S12).

Genetic Cascades Increase the Variability of Gene Expression. We then explored the effect of a simple genetic cascade on the variability of protein expression. This cascade consisted of the expression of T3 RNA polymerase from a T7 transcriptional promoter followed by the expression of RFP from a T3 transcriptional promoter (Figure 2e). The computational model was updated to include the new parameters (e.g., the kinetics of T3 RNA polymerase) (Tables S9-S11). Thirteen different constructs containing three different $\mathrm{T} 7$ transcriptional promoters and three different ribosome binding sites were selected. The model predicted that increased expression of RFP was mainly due to two elements: a medium-to-weak strength $\mathrm{T} 7$ transcriptional promoter and a strong RBS for RFP (Figure S13). By modifying the noise parameters according to their estimated probability distribution, the model did not predict a significant increase in the coefficient of variation for the synthesis of protein in the cascade reaction with respect to the expression of the single gene. The 13 constructs were then tested in the laboratory in two different ways. The two genes of the cascade were either placed on separate pieces of DNA or together on the same DNA template. Most cell-free genetic circuitry reported in the a)

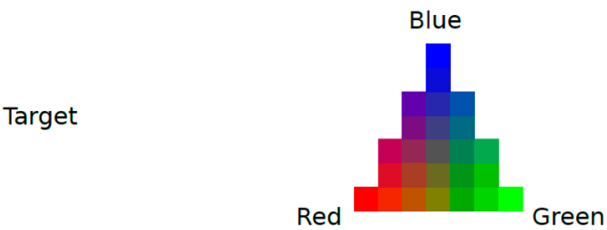

b)

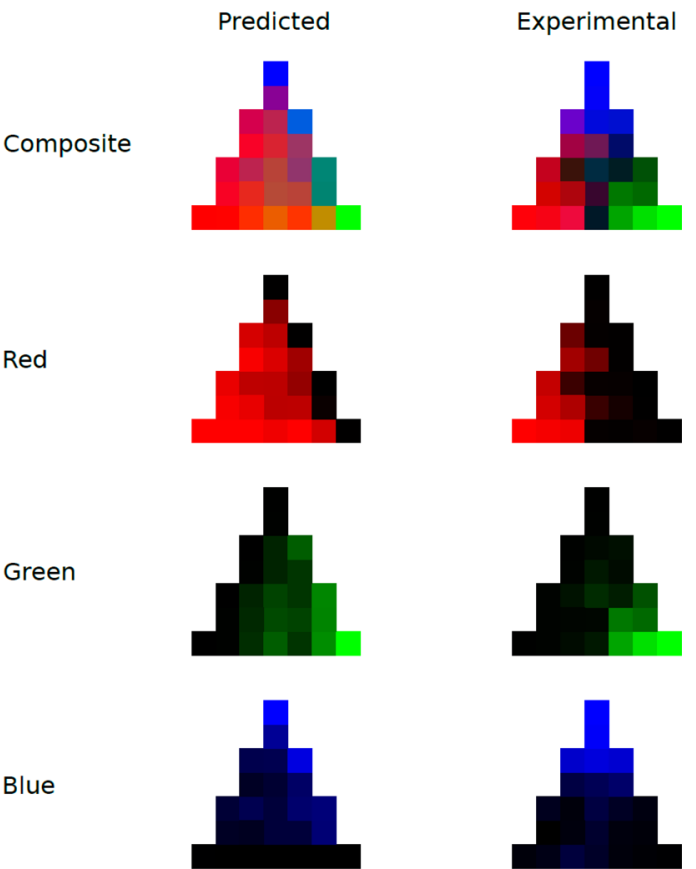

Figure 3. Transcription-translation reactions can be used to construct an RGB color triangle. (a) The target picture is composed of 25 pixels, each displaying a different color resulting from the three different gradients that stem from the vertexes of the triangle. (b) The computational model was employed to decide which combination of promoters and ribosome binding sites should be used to achieve the desired color for each pixel (Figure S14, Table S12). On the basis of the computational prediction for the expression of each gene in each pixel, a predicted image was generated. Each sequence was then assembled and run.

literature exploits separate pieces of DNA to accelerate the screening of biological parts; however, the effect of such architecture on expression and variability are unexplored. Contrary to what the model predicted, the coefficient of variation increased by 120 and $70 \%$ for RNA and protein synthesis, respectively, for the expression of the cascade in comparison to that of a single gene for reactions run with a single batch of transcription-translation machinery and using a single DNA template (Figure 2c). The analogous increase in the coefficient of variation for experiments run with multiple batches was 130 and $43 \%$ for RNA and protein synthesis, respectively. The fact that the coefficient of variation significantly increased when going from a single gene construct to a simple, two-step cascade suggests that genetic circuitry of increased complexity will concomitantly increase the variability of expression. Furthermore, unlike the single gene data, this specific cascade showed variability in the expression of the final RNA of the cascade that was similar to that of the final protein. This is likely because the first gene of the cascade expressed the RNA polymerase needed to transcribe the second gene. In 
other words, the variability associated with producing the $\mathrm{T} 3$ RNA polymerase directly impacted the extent of transcription of the second gene. The coefficient of variation of the cascade consisting of two pieces of DNA run with multiple batches of transcription-translation machinery was large, so care should be taken in screening genetic circuits with multiple pieces of DNA in vitro. The increase in variability of expressed RNA and protein from the cascade was visually confirmed by the construction of a yin-yang image (Figure S15). Finally, the genetic cascade also revealed the existence of the influence of depleting resources. Transcription and translation share common reactants, ${ }^{9}$ and so some degree of competition for resources was expected although not previously observed for noncascading reactions. ${ }^{16}$ For example, the use of strong transcriptional promoters and ribosome binding sites resulted in relatively low amounts of RFP (Figure S16). The configuration that produced the most RFP contained a weak T7 transcriptional promoter, a medium strength RBS for T3 RNA polymerase, and a strong RBS for RFP, which was consistent with the kinetic model for the cascade.

Because the inclusion of a transcriptional repressor can be exploited to confer sensing capability on artificial cells ${ }^{5,31}$ and potentially on cascading cell-free networks, we next sought to investigate the behavior of genetic constructs encoding a transcriptional repressor. This construct contained two T7 transcriptional promoters and two ribosome binding sites (Figure 2e). Each transcriptional promoter and RBS pair controlled the expression of a single gene, either RFP or the transcriptional repressor. The region upstream of the RFP encoding sequence also contained an operator sequence to bind the transcriptional repressor. A LuxR homologue, D91G $\mathrm{EsaR}^{32}$ (hereafter referred to as EsaR), from the organism Pantoea stewartii was used as the transcriptional repressor. In the absence of EsaR's cognate ligand, EsaR blocks transcription, and in the presence of the quorum signal 3-oxohexanoylhomoserine lactone (3OC6HSL), expression is derepressed.

After screening a small set of different spacer sequences between the transcriptional promoter and the operator sequence (Table S13, Figure S17), a kinetic model for protein expression under the control of EsaR was built and estimated (Tables S14-S16). Eight different genetic constructs that the model predicted to show a gradient of expression between repressed and derepressed states were assembled onto a single DNA (Table S17). Of these genetic constructs, four showed at least 5-fold derepression of transcription in the presence of 3 OC6HSL, and four gave at least 2-fold more cell-free expressed protein when 3OC6HSL was added to the reaction (Figure S18). The transcription and translation data correlated well with the predictions from the computational model $(0.74$ and 0.87 for RNA and RFP expression, respectively) even if the model predicted higher absolute concentrations. When rescaled to the actual observed concentrations, the expression ratios matched well the experimental data (Figure S19). However, because little RNA is needed to make much protein, ${ }^{16}$ none of the constructs yielded low RFP concentration in the absence of 3OC6HSL and high RFP concentration in the presence of 3OC6HSL. Altering the DNA template concentration from 1.6 to $0.42 \mathrm{nM}$ did not significantly impact the difference between repressed and derepressed states (Figure S20). Nevertheless, clear on-off switching activity was observed. The coefficient of variation of expressed protein was similar to the transcriptiontranslation of single gene constructs (Figure 2). The difference was again with the RNA, which was similar to that of the protein. This similarity in variability of expressed RNA and protein was observed with both genetic cascades, the cascade in which the first gene encoded a RNA polymerase and the cascade in which the first gene encoded a transcriptional repressor. In both cases, the variability in the synthesis of the product of the first gene directly impacted the extent of transcription of the second gene.

A Better Understanding of the Influence of RNA Folding on Transcription-Translation May Improve Predictability. It has often been argued that biological parts need to be thoroughly characterized so that a set of rules and equations can be formulated that would allow for the construction of more complex systems with predictable behavior. $^{33,34}$ However, to date this approach has not been successful in vivo or in vitro. Even in the simplified case of the cell-free transcription-translation of a single gene, the influence of the RBS is not consistent. The inconsistency in the activity of the RBS likely reflects a dependence on the entire sequence of the mRNA. That is, different sequences fold differently, which affects the accessibility of the RBS, the initiation of translation, ${ }^{35}$ and ribosome density. ${ }^{36}$ To better probe whether RNA folding impacted the cell-free expression of protein, four different genetic sequences encoding BFP were tested. The original BFP sequence, which was codon optimized for yeast and bacteria, was resynthesized to maintain the same amino acid sequence but to have maximal or minimal GC content, and an additional sequence was made that was specifically codon optimized for E. coli. From these four sequences, the GC content appeared to strongly impact the amount of synthesized protein but not the RNA concentration (Figure S21). For the effect on translation to be isolated, the protein concentration was divided by the RNA concentration, revealing a clear correlation between higher GC content with the increased expression of protein (Figure 4). These data are similar to those

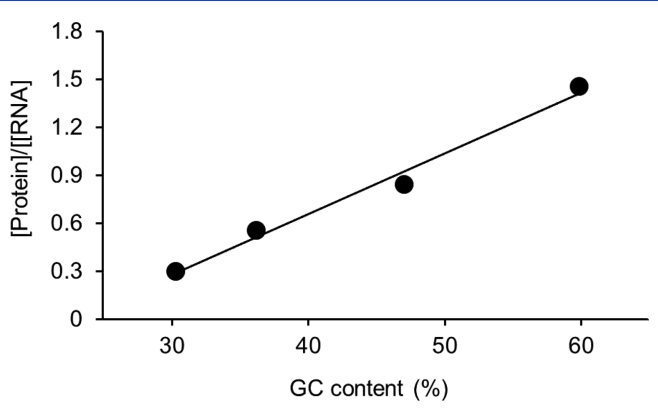

Figure 4. GC content of the coding sequence of BFP affects the synthesis of protein. The coding sequence of the protein was modified to maximize either the AT or GC content. Additionally, a codonoptimized version of the coding sequence was generated to assess the impact on translation. Each construct was expressed with the PURE system. The DNA constructs from lower GC content to higher GC content were NG046 (AT-rich, GC content: 30.4\%), NG045 (original sequence, GC content: 36.3\%), NG047 (codon-optimized, GC content: $47.1 \%$ ), and NG048 (GC-rich, GC content: $60.0 \%$ ). $R^{2}=$ 0.985 .

observed previously in vivo in Saccharomyces cerevisiae. ${ }^{37}$ The mechanism of this effect is not known, but increased GC content is associated with an increased density of ribosomes on mRNA. Although similar studies have not been performed with bacteria, a bias for higher GC content codons is found across all three Kingdoms of life. ${ }^{38}$ More work is needed to determine how robust the relationship between GC content and protein 
expression is with bacterial systems. Nevertheless, it does appear that mRNA folding significantly affects the expression of protein. Taken together with previous studies on the initiation of translation, ${ }^{39}$ high protein expression may be best achieved with sequences of low structure around the RBS, including the 13 codons after the start $\operatorname{codon}^{35}$ and high GC content throughout the remainder of the gene.

The RBS is not the only complicating factor in predicting the expression of protein. Variability is always present due to the intrinsic stochastic nature of basic molecular events, such as transcription and translation. The stochasticity of compartmentalized systems has been evaluated, ${ }^{22,23,40}$ but the variability of bulk transcription-translation reactions have not been thoroughly investigated. Some of the noise observed in encapsulated, cell-free gene expression was thought to either arise from differences in DNA template copy number across different droplets or from the intrinsic stochastic nature of transcription itself. ${ }^{22,23}$ In bulk, where complications arising from encapsulation do not exist, transcription was not found to exhibit much variability. However, variability in transcription significantly increased when different genes of a network were encoded within different strands of DNA. Having genes on separate DNA strands likely introduced variability in the ratio of templates in a somewhat similar way to the influence of DNA template copy number on transcription encapsulated within droplets. In bulk reactions, protein synthesis provided the largest source of variability, which likely reflected in part the complexity of translation and the consequences of using a template that can assume a variety of structures with different degrees of stability. Furthermore, the variability associated with translation is confounded by the batch-to-batch variability of the transcription-translation machinery used to mediate gene expression.

Current computational models do not adequately take into consideration RNA folding. Thus far, models are built by incorporating a data set containing the activity of biological parts, and these resulting models can usually accurately describe the behavior of those same biological parts under previously tested conditions. However, much more is needed if these models are to be of real engineering value. It is not sufficient to describe and understand a system. Instead, within a certain margin of error, the behavior of a new device, potentially under new conditions, needs to be reliably predicted; otherwise, the construction of new genetic devices remains a process of trial and error. Unfortunately, the development of a model that fits the experimentally available data does not necessarily mean that the model is capable of predicting experiments that have yet to be run, i.e., there is a difference between model estimation and prediction. For example, the model used herein can predict the expression of each protein individually, but the data set for the expression of RFP cannot be used to reliably predict the expression of GFP. It seems that what is missing is a deeper understanding of the role of RNA. A step forward in this direction was the development of an equilibrium statistical thermodynamic model to predict the activity of an RBS sequence within the context of the surrounding RNA. ${ }^{41}$ However, the dynamics governing the folding of mRNA and the relationship between the kinetics of mRNA folding and gene expression are still not sufficiently understood. ${ }^{42}$ Therefore, as noted before, ${ }^{43,44}$ there is approximately a $50 \%$ chance that the predictions will fall within 2-fold of experimentally determined protein levels. For now, it may be wise to simply exploit tools that incorporate the folding of the RNA, as in the
RBS calculator, and the variability in gene expression to identify a restricted set of candidate sequences for screening in the laboratory.

\section{METHODS}

Genetic Constructs. Genes encoding the fluorescent proteins GFPmut3b and mRFP1 and the Spinach aptamer were from previously described constructs. ${ }^{16}$ Genetic sequence of the T3 RNA polymerase (BBa_K346000) was from the registry of standard biological parts (http://partsregistry.org). Genes encoding the proteins Azurite (referred to as BFP) and D91G EsaR were from Addgene (plasmids \#14034 and \#47646, respectively). All other genes were synthesized by Eurofins Scientific. All genes were subcloned into pET21b by isothermal Gibson assembly. ${ }^{45}$ All constructs were confirmed by sequencing by GATC Biotech.

Cell-Free Transcription-Translation. Unless otherwise indicated, $9 \mu \mathrm{L}$ transcription-translation reactions with the PURExpress in vitro protein synthesis kit (New England Biolabs) contained $12.6 \mathrm{nM}$ of linear DNA template and 4 units of human placenta RNase inhibitor (New England BioLabs). When needed, DFHBI (Lucerna) was added to a final concentration of $60 \mu \mathrm{M}$. The reaction components were assembled in an ice-cold metal plate, and the reaction was initiated by incubation at $37^{\circ} \mathrm{C}$. Reactions were monitored for $6 \mathrm{~h}$ with a Rotor-Gene Q 6plex system (Qiagen). The blue channel was used to detect Azurite (excitation: $365 \pm 20 \mathrm{~nm}$; emission: $460 \pm 20 \mathrm{~nm}$ ); the green channel was used to detect GFPmut $3 \mathrm{~b}$ and Spinach (excitation: $470 \pm 10 \mathrm{~nm}$; emission: $510 \pm 5 \mathrm{~nm})$, and the orange channel was used to detect mRFP1 (excitation: $585 \pm 5 \mathrm{~nm}$; emission: $610 \pm 5 \mathrm{~nm}$ ). Each reaction was repeated at least three times. The template DNA concentration was $0.5 \mathrm{nM}$ for single gene expression experiments. The cascade genetic circuit with the two genes on two separate pieces of DNA employed the following template concentrations: $12.6 \mathrm{nM}$ (reporter gene encoding mRFP1 and Spinach) and $4.2 \mathrm{nM}$ (gene coding for T3 RNA polymerase). The cascade genetic circuit with two genes on the same piece of DNA employed a template DNA concentration of $5 \mathrm{nM}$. Finally, the experiments performed with the repressible genetic circuit employed a template DNA concentration of $5 \mathrm{nM}$. When used, the concentration of 3-oxohexanoyl-homoserine lactone was $5 \mu \mathrm{M}$.

Cell-Free Extract Preparation. E. coli Rosetta 2(DE3) was grown in $2 \times \mathrm{YTP}$ media supplemented with $40 \mathrm{mM} \mathrm{K}_{2} \mathrm{HPO}_{4}$, $22 \mathrm{mM} \mathrm{KH}_{2} \mathrm{PO}_{4}$, and $34 \mu \mathrm{g} / \mathrm{mL}$ of chloramphenicol overnight at $37^{\circ} \mathrm{C}$ and $220 \mathrm{rpm}$. Next, an aliquot was diluted to $\mathrm{OD}^{600 \mathrm{~nm}}$ $=0.01$ and incubated for $3.5 \mathrm{~h}$ at $37{ }^{\circ} \mathrm{C}$ and $220 \mathrm{rpm}$. Cells were then pelleted at $6000 \mathrm{~g}$ for $6 \mathrm{~min}$ at $4{ }^{\circ} \mathrm{C}$. The extract was then prepared closely following the protocol from the Noireaux laboratory ${ }^{46}$ with the following exceptions: Snakeskin dialysis membranes (10 kDa MWCO Thermo fischer-68100) were used in place of the Slide-A-Lyzer dialysis cassettes for $3 \mathrm{~h}$ at 4 ${ }^{\circ} \mathrm{C}$. Aliquots of freshly prepared extract were flash-frozen with liquid nitrogen and stored at $-80{ }^{\circ} \mathrm{C}$. The amino acid solution was prepared following the indications of Cashera et al. for equimolar concentrations at $\mathrm{pH} 7.86 .^{47}$ The composition of the cell-free reaction was $10 \mathrm{nM}$ plasmid DNA, 33\% (v/v) cell-free extract, $7.14 \%(\mathrm{v} / \mathrm{v})$ energetic solution, $1.5 \mathrm{mM}$ amino acid solution, and $2 \%(\mathrm{v} / \mathrm{v})$ PEG-8000. The reactions were supplemented with $12 \mathrm{mM}$ maltose for ATP regeneration ${ }^{48}$ and $5 \mathrm{mM}$ or $10 \mathrm{mM} \mathrm{Mg}^{2+}$-glutamate for reactions containing T7 RNA polymerase ${ }^{49}$ and the endogenous E. coli RNA 
polymerase, respectively. For mRNA to be monitored through the encoded Spinach aptamer, reactions contained $60 \mu \mathrm{M}$ DFHBI (Lucerna), resulting in a final DMSO concentration of $0.4 \%(\mathrm{v} / \mathrm{v})$.

Genetically Encoded Pictures. Pixelated images (14×14) were generated with four different DNA templates. Following the target picture, the number of different $9 \mu \mathrm{L}$ PURE system reactions required to fill the pixels was calculated. Reactions were set up with components from different batches of the PURE system. After $6 \mathrm{~h}$ of incubation at $37{ }^{\circ} \mathrm{C}$, each reaction was pipetted into the wells of the plate (ViewPlate-1536 F from PerkinElmer) to produce the pixelated image. After filling all of the required wells, the plate was centrifuged at $4000 \mathrm{rpm}$ for 1 min at $4{ }^{\circ} \mathrm{C}$ with a Thermo Scientific Legend X1R centrifuge with a T20 microplate rotor. A Typhoon Trio from GE Healthcare was used to visualize the picture. For the RNA picture, a blue laser was used $(488 \mathrm{~nm})$ for excitation in combination with a 526 short-pass filter (SP filter transmitting light below $526 \mathrm{~nm}$ ) for emission. For the protein picture, a green laser was used $(532 \mathrm{~nm})$ for excitation in combination with the 610 BP 30 filter (transmitting light between 595 and $625 \mathrm{~nm}$ ) for emission. For both of the pictures, the gain was set to $1000 \mathrm{~V}$.

For the multicolored triangle picture, a 384-well plate (Greiner Bio-one 384 Flat Bottom Black) was employed. Each well, representing a single pixel of the picture, was filled with a $9 \mu \mathrm{L}$ PURE system reaction. Each reaction included three different DNA templates encoding the fluorescent proteins mRFP1, GFPmut3b, and Azurite, each controlled by the appropriate combination of promoter and ribosome binding site. The reaction was incubated in a PCR tube at 37 ${ }^{\circ} \mathrm{C}$ for $6 \mathrm{~h}$, after which each reaction was placed into the corresponding well of the 384-well plate. Next, the plate reader Tecan Infinite 200 was used to record the fluorescence of Azurite $\left(\lambda_{\mathrm{ex}}=377 \mathrm{~nm}, \lambda_{\mathrm{em}}=472 \mathrm{~nm}\right)$, GFPmut $3 \mathrm{~b}\left(\lambda_{\mathrm{ex}}=474\right.$ $\left.\mathrm{nm}, \lambda_{\mathrm{em}}=511 \mathrm{~nm}\right)$, and $\mathrm{mRFP}\left(\lambda_{\mathrm{ex}}=579 \mathrm{~nm}, \lambda_{\mathrm{em}}=613 \mathrm{~nm}\right)$. The excitation bandwidth was $9 \mathrm{~nm}$, and the emission bandwidth was $20 \mathrm{~nm}$. The gain was set to 115 . The image generated with the repressor-encoding genetic cascade was additionally collected with the Spinach aptamer $\left(\lambda_{\mathrm{ex}}=469 \mathrm{~nm}\right.$, $\left.\lambda_{\text {em }}=501 \mathrm{~nm}\right)$.

Protein and RNA Standard Curves. Standard curves to translate fluorescence intensity into molar concentrations were generated by using recombinantly expressed and purified fluorescent proteins. His-tagged versions of GFPmut3b, Azurite, and mRFP1 were generated by mutating the stop codon within $\mathrm{pET} 21 \mathrm{~b}$. The resulting constructs contained an additional 24 residues including a carboxy-terminal hexahistidine tag. Each His-tagged construct was purified as described previously. ${ }^{16}$ Similarly, purified RNA harboring the Spinach aptamer and transcribed from the different constructs was used to equate the fluorescence intensity of the Spinach aptamer with molar concentrations. Transcription reactions were assembled and then purified as previously described. ${ }^{16}$

Computational Kinetic Model. COPASI ${ }^{50}$ was used to implement a computational model that consisted of 10 reactions and 12 parameters describing cell-free RNA and protein expression (Figure S10, Tables S6-S8). The resources necessary for transcription and translation were modeled as a single species ( $\mathrm{R} 1$ and $\mathrm{R} 2$, respectively) and were subject to degradation. Here, resources refer not only to the molecular machinery required to sustain transcription and translation, such as the T7 RNA polymerase, ribosomes, and all accessory protein factors, but also to the small molecules required to sustain each reaction. Following the PURE system composition, ${ }^{17}$ only the initial concentration of T7 RNA polymerase and ribosomes were defined. For the rest of the species and for the reaction rates, parameter estimations based on experimental data were performed. Parameter estimations were also included for two noise parameters, including a $\pm 5 \%$ range for the template DNA concentration and a $\pm 10 \%$ range for the composition of the PURE system.

Statistical Analysis. The coefficient of variation was calculated as the ratio between the standard deviation and the mean value of each experiment $(\mathrm{CV}=\sigma / \mu)$. The coefficient of variation for single batch experiments was calculated by averaging the coefficients of variation of three replicates, each collected with the same batch of the PURE system. The coefficient of variation for multiple batch experiments was calculated by averaging the coefficients of variation of nine replicates using three different PURE system batches (three replicates per batch).

Photobleaching. Twenty microliters of $2.5 \mu \mathrm{M}$ RFP, 2.5 $\mu \mathrm{M}$ BFP, and $2.5 \mu \mathrm{M}$ GFP purified protein solutions in $10 \mathrm{mM}$ Tris- $\mathrm{HCl}$ at $\mathrm{pH} 7.5$ were loaded into a Greiner Flat Bottom Black Polystyrene 384-well plate (preheated for $10 \mathrm{~min}$ at 37 $\left.{ }^{\circ} \mathrm{C}\right)$. A Tecan Infinite PRO200 plate reader was used to record the fluorescence of the single proteins with their specific excitation settings followed by measurements at each of the following wavelengths in the order listed: blue $\left(\lambda^{\mathrm{ex}}=377 \mathrm{~nm}\right.$, $\left.\lambda^{\mathrm{em}}=472 \mathrm{~nm}\right)$, red $\left(\lambda^{\mathrm{ex}}=579 \mathrm{~nm}, \lambda^{\mathrm{em}}=613 \mathrm{~nm}\right)$, green $\left(\lambda^{\mathrm{ex}}=\right.$ $\left.474 \mathrm{~nm}, \lambda^{\mathrm{em}}=511 \mathrm{~nm}\right)$. The excitation bandwidth was $9 \mathrm{~nm}$, and the emission bandwidth was $20 \mathrm{~nm}$. The gain was set to 115 .

\section{ASSOCIATED CONTENT}

\section{Supporting Information}

The Supporting Information is available free of charge on the ACS Publications website at DOI: 10.1021/acssynbio.6b00250.

Additional tables and figures based on descriptions provided in the text as well as links to the deposited genetic sequences in the ACS Synthetic Biology Registry (PDF)

\section{AUTHOR INFORMATION}

\section{Corresponding Author}

*E-mail:mansy@science.unitn.it.

ORCID

Sheref S. Mansy: 0000-0003-2382-198X

Notes

The authors declare no competing financial interest.

\section{ACKNOWLEDGMENTS}

We thank the Armenise-Harvard foundation and CIBIO for funding, Cristina Del Bianco of the protein technology facility at CIBIO for assistance, and Federico Brunello for help with cloning and the initial characterization of BFP. This research was funded in part by the Autonomous Province of Trento, "Grandi Progetti 2012", project "Characterizing and improving brain mechanisms of attention-ATTEND”.

\section{REFERENCES}

(1) Spirin, A. S., and Swartz, J. R. (2008) Cell-free protein synthesis: methods and protocols, Wiley-VCH. 
(2) Hansen, M. M. K., Meijer, L. H. H., Spruijt, E., Maas, R. J. M., Rosquelles, M. V., Groen, J., Heus, H. A., and Huck, W. T. S. (2015) Macromolecular crowding creates heterogeneous environments of gene expression in picolitre droplets. Nat. Nanotechnol. 11, 191-197.

(3) Jewett, M. C., and Forster, A. C. (2010) Update on designing and building minimal cells. Curr. Opin. Biotechnol. 21, 697-703.

(4) Terasaka, N., and Suga, H. (2014) Flexizymes-facilitated Genetic Code Reprogramming Leading to the Discovery of Drug-like Peptides. Chem. Lett. 43, 11-19.

(5) Lentini, R., Yeh Martín, N., and Mansy, S. S. (2016) Communicating artificial cells. Curr. Opin. Chem. Biol. 34, 53-61.

(6) Olins, P. O., Devine, C. S., Rangwala, S. H., and Kavka, K. S. (1988) The T7 phage gene 10 leader RNA, a ribosome-binding site that dramatically enhances the expression of foreign genes in Escherichia coli. Gene 73, 227-235.

(7) Siegal-Gaskins, D., Tuza, Z. A., Kim, J., Noireaux, V., and Murray, R. M. (2014) Gene circuit performance characterization and resource usage in a cell-free "breadboard. ACS Synth. Biol. 3, 416-425.

(8) Stogbauer, T., Windhager, L., Zimmer, R., and Radler, J. O. (2012) Experiment and mathematical modeling of gene expression dynamics in a cell-free system. Integr. Biol. 4, 494-501.

(9) Calviello, L., Stano, P., Mavelli, F., Luisi, P. L., and Marangoni, R. (2013) Quasi-cellular systems: stochastic simulation analysis at nanoscale range. BMC Bioinformatics 14 (Suppl 7), S7.

(10) Karzbrun, E., Shin, J., Bar-Ziv, R. H., and Noireaux, V. (2011) Coarse-Grained Dynamics of Protein Synthesis in a Cell-Free System. Phys. Rev. Lett. 106, 048104.

(11) Tuza, Z. A., Singhal, V., Jongmin Kim, J., and Murray, R. M. (2013) An in silico modeling toolbox for rapid prototyping of circuits in a biomolecular "breadboard" system, in 52nd IEEE Conference on Decision and Control, IEEE, pp 1404-1410.

(12) Nielsen, A. A. K., Der, B. S., Shin, J., Vaidyanathan, P., Paralanov, V., Strychalski, E. A., Ross, D., Densmore, D., and Voigt, C. A. (2016) Genetic circuit design automation. Science 352, 7341.

(13) Kosuri, S., Goodman, D. B., Cambray, G., Mutalik, V. K., Gao, Y., Arkin, A. P., Endy, D., and Church, G. M. (2013) Composability of regulatory sequences controlling transcription and translation in Escherichia coli. Proc. Natl. Acad. Sci. U. S. A. 110, 14024-14029.

(14) Temme, K., Zhao, D., and Voigt, C. A. (2012) Refactoring the nitrogen fixation gene cluster from Klebsiella oxytoca. Proc. Natl. Acad. Sci. U. S. A. 109, 7085-7090.

(15) Lentini, R., Forlin, M., Martini, L., Del Bianco, C., Spencer, A. C., Torino, D., and Mansy, S. S. (2013) Fluorescent Proteins and in Vitro Genetic Organization for Cell-Free Synthetic Biology. ACS Synth. Biol. 2, 482-489.

(16) Chizzolini, F., Forlin, M., Cecchi, D., and Mansy, S. S. (2014) Gene position more strongly influences cell-free protein expression from operons than T7 transcriptional promoter strength. ACS Synth. Biol. 3, 363-371.

(17) Shimizu, Y., Inoue, A., Tomari, Y., Suzuki, T., Yokogawa, T., Nishikawa, K., and Ueda, T. (2001) Cell-free translation reconstituted with purified components. Nat. Biotechnol. 19, 751-5.

(18) Zelcbuch, L., Antonovsky, N., Bar-Even, A., Levin-Karp, A., Barenholz, U., Dayagi, M., Liebermeister, W., Flamholz, A., Noor, E., Amram, S., Brandis, A., Bareia, T., Yofe, I., Jubran, H., and Milo, R. (2013) Spanning high-dimensional expression space using ribosomebinding site combinatorics. Nucleic Acids Res. 41, e98.

(19) Mutalik, V. K., Guimaraes, J. C., Cambray, G., Mai, Q.-A., Christoffersen, M. J., Martin, L., Yu, A., Lam, C., Rodriguez, C., Bennett, G., Keasling, J. D., Endy, D., and Arkin, A. P. (2013) Quantitative estimation of activity and quality for collections of functional genetic elements. Nat. Methods 10, 347-53.

(20) Karig, D. K., Iyer, S., Simpson, M. L., and Doktycz, M. J. (2012) Expression optimization and synthetic gene networks in cell-free systems. Nucleic Acids Res. 40, 3763-74.

(21) Chappell, J., Jensen, K., and Freemont, P. S. (2013) Validation of an entirely in vitro approach for rapid prototyping of DNA regulatory elements for synthetic biology. Nucleic Acids Res. 41, 34713481 .
(22) Nishimura, K., Tsuru, S., Suzuki, H., and Yomo, T. (2015) Stochasticity in Gene Expression in a Cell-Sized Compartment. ACS Synth. Biol. 4, 566-576.

(23) Karig, D. K., Jung, S. Y., Srijanto, B., Collier, C. P., and Simpson, M. L. (2013) Probing cell-free gene expression noise in femtoliter volumes. ACS Synth. Biol. 2, 497-505.

(24) Pukelsheim, F. (1993) Optimal design of experiments, Wiley$\mathrm{VCH}$, New York.

(25) Hansen, M. M. K., Ventosa Rosquelles, M., Yelleswarapu, M., Maas, R. J. M., van Vugt-Jonker, A. J., Heus, H. a., and Huck, W. T. S. (2016) Protein Synthesis in Coupled and Uncoupled Cell-Free Prokaryotic Gene Expression Systems. ACS Synth. Biol. 5, 1433-1440.

(26) Mutalik, V. K., Guimaraes, J. C., Cambray, G., Lam, C., Christoffersen, M. J., Mai, Q.-A., Tran, A. B., Paull, M., Keasling, J. D., Arkin, A. P., and Endy, D. (2013) Precise and reliable gene expression via standard transcription and translation initiation elements. Nat. Methods 10, 354-60.

(27) Chevance, F. F. V, Le Guyon, S., and Hughes, K. T. (2014) The Effects of Codon Context on In Vivo Translation Speed. PLoS Genet. 10, e1004392.

(28) Vasquez, J. R., Evnin, L. B., Higaki, J. N., and Craik, C. S. (1989) An expression system for trypsin. J. Cell. Biochem. 39, 265-76.

(29) Hayashi, M. N., and Hayashi, M. (1985) Cloned DNA sequences that determine mRNA stability of bacteriophage ФX174 in vivo are functional. Nucleic Acids Res. 13, 5937-5948.

(30) Salis, H. M. (2011) The ribosome binding site calculator. Methods Enzymol. 498, 19-42.

(31) Lentini, R., Santero, S. P., Chizzolini, F., Cecchi, D., Fontana, J., Marchioretto, M., Del Bianco, C., Terrell, J. L., Spencer, A. C., Martini, L., Forlin, M., Assfalg, M., Dalla Serra, M., Bentley, W. E., and Mansy, S. S. (2014) Integrating artificial with natural cells to translate chemical messages that direct E. coli behaviour. Nat. Commun. 5, 4012.

(32) Shong, J., Huang, Y.-M., Bystroff, C., and Collins, C. H. (2013) Directed Evolution of the Quorum-Sensing Regulator EsaR for Increased Signal Sensitivity. ACS Chem. Biol. 8, 789-795.

(33) Ellis, T., Adie, T., and Baldwin, G. S. (2011) DNA assembly for synthetic biology: from parts to pathways and beyond. Integr. Biol. 3, 109.

(34) Purnick, P. E. M., and Weiss, R. (2009) The second wave of synthetic biology: from modules to systems. Nat. Rev. Mol. Cell Biol. $10,410-422$.

(35) Borujeni, A. E., Cetnar, D., Farasat, I., Smith, A., Lundgren, N., and Salis, H. M. (2016) Precise Quantification of Translation Inhibition by mRNA Structures that Overlap with the Ribosomal Footprint in N-terminal Coding Sequences. bioRxiv, 91470.

(36) Mao, Y., Liu, H., Liu, Y., and Tao, S. (2014) Deciphering the rules by which dynamics of mRNA secondary structure affect translation efficiency in Saccharomyces cerevisiae. Nucleic Acids Res. $42,4813-4822$.

(37) Zur, H., and Tuller, T. (2012) Strong association between mRNA folding strength and protein abundance in S. cerevisiae. EMBO Rep. 13, 272-277.

(38) Knight, R. D., Freeland, S. J., and Landweber, L. F. (2001) A simple model based on mutation and selection explains trends in codon and amino-acid usage and GC composition within and across genomes. Genome Biol. 2, 1-13.

(39) Kudla, G., Murray, A. W., Tollervey, D., and Plotkin, J. B. P. (2009) Coding-Sequence Determinants of Gene Expression in Escherichia coli. Science (Washington, DC, U. S.) 324, 255-258.

(40) Hansen, M. M. K., Meijer, L. H. H., Spruijt, E., Maas, R. J. M., Rosquelles, M. V., Groen, J., Heus, H. A., and Huck, W. T. S. (2015) Macromolecular crowding creates heterogeneous environments of gene expression in picolitre droplets. Nat. Nanotechnol. 11, 191-197.

(41) Salis, H. M., Mirsky, E. A., and Voigt, C. A. (2009) Automated design of synthetic ribosome binding sites to control protein expression. Nat. Biotechnol. 27, 946-950.

(42) Espah Borujeni, A., Channarasappa, A. S., and Salis, H. M. (2014) Translation rate is controlled by coupled trade-offs between 
site accessibility, selective RNA unfolding and sliding at upstream standby sites. Nucleic Acids Res. 42, 2646-59.

(43) Whitaker, W. R., Lee, H., Arkin, A. P., and Dueber, J. E. (2015) Avoidance of Truncated Proteins from Unintended Ribosome Binding Sites within Heterologous Protein Coding Sequences. ACS Synth. Biol. 4, 249-257.

(44) Wang, Y.-H., Wei, K. Y., and Smolke, C. D. (2013) Synthetic biology: advancing the design of diverse genetic systems. Annu. Rev. Chem. Biomol. Eng. 4, 69-102.

(45) Gibson, D. G., Young, L., Chuang, R.-Y., Venter, J. C., Hutchison, C. A., and Smith, H. O. (2009) Enzymatic assembly of DNA molecules up to several hundred kilobases. Nat. Methods 6, 3435 .

(46) Sun, Z. Z., Hayes, C. A., Shin, J., Caschera, F., Murray, R. M., and Noireaux, V. (2013) Protocols for implementing an Escherichia coli based TX-TL cell-free expression system for synthetic biology. J. Visualized Exp., e50762.

(47) Caschera, F., and Noireaux, V. (2015) Preparation of amino acid mixtures for cell-free expression systems. BioTechniques 58, 40-43.

(48) Caschera, F., and Noireaux, V. (2014) Synthesis of $2.3 \mathrm{mg} / \mathrm{mL}$ of protein with an all Escherichia coli cell-free transcription-translation system. Biochimie 99, 162-8.

(49) Ellinger, T., and Ehricht, R. (1998) Single-step purification of T7 RNA polymerase with a 6-histidine tag. Biotechniques 24, 718-720. (50) Hoops, S., Sahle, S., Gauges, R., Lee, C., Pahle, J., Simus, N., Singhal, M., Xu, L., Mendes, P., and Kummer, U. (2006) COPASI-a COmplex PAthway SImulator. Bioinformatics 22, 3067-3074. 